\title{
Bazo errante infartado y vólvulo de cola de páncreas en un paciente con hernia de Grynfelt-Lesshaft concomitante
}

\author{
Infarcted wandering spleen and pancreatic volvulus in a patient with concomitant \\ Grynfelt-Lesshaft haernia
}

\author{
Daniel E. Saldívar-Martínez, Héctor M. Galindo-Sánchez*, Ignacio Fonseca-Sada, \\ Edson R. Marcos-Ramírez y Francisco Vázquez-Fernández \\ Departamento de Cirugía General, Hospital Universitario Dr. José Eleuterio González, Monterrey, Nuevo León, México
}

\begin{abstract}
Resumen
El bazo errante es una afección rara que en general se debe a anomalías congénitas del mesogastrio dorsal o de los ligamentos suspensores esplénicos, y no a traumatismos. La complicación más frecuente es la torsión del hilio esplénico con isquemia o infarto esplénico, y su asociación a vólvulo pancreático es extremadamente rara. No existen ningún otro caso reportado de bazo errante con vólvulo pancreático en un paciente que presenta una hernia lumbar de Grynfelt-Lesshaft postraumática. Presentamos el caso de una paciente de 43 años con asociación de estas tres afecciones y antecedente de traumatismo abdominal.
\end{abstract}

Palabras clave: Bazo errante. Infarto esplénico. Vólvulo pancreático. Hernia lumbar. Grynfelt-Lesshaft.

\begin{abstract}
Wandering spleen is a rare condition, generally due to congenital abnormalities of the dorsal mesogastrium or splenic suspensory ligaments and not traumatic events. The most frequent complication is torsion of the splenic hilus with ischemia or splenic infarction and its association with pancreatic volvulus is extremely rare. There are no reported cases of wandering spleen with pancreatic volvulus in association with a post traumatic Grynfelt-Lesshaft haernia. We present a case of a 43-yearold female patient with an association of these three entities and a history of abdominal trauma.
\end{abstract}

Key words: Wandering spleen. Splenic infarction. Pancreatic volvulus. Lumbar haernia. Grynfelt-Lesshaft.

\section{Introducción}

El bazo errante se define como un bazo fuera de su posición habitual o en un estado ptósico, generalmente hacia el abdomen inferior ${ }^{1}$, debido a anomalías congénitas en los ligamentos suspensores esplénicos. Tiene una incidencia inferior al $0.2 \%$ en pacientes sometidos a esplenectomía1. Su complicación más frecuente es la torsión del hilio esplénico, con la consecuente isquemia que lleva a infarto esplénico. E

\section{Correspondencia:}

*Héctor M. Galindo-Sánchez

Bolivia 435

Col. Vista Hermosa

Fecha de recepción: 23-07-2020

C.P. 64620 , Monterrey, N.L., México

E-mail: hector.galindo8@gmail.com 0009-7411/@ 2020 Academia Mexicana de Cirugía. Publicado por Permanyer. Este es un artículo open access bajo la licencia CC BY-NC-ND (http://creativecommons.org/licenses/by-nc-nd/4.0/).

Cir Cir. 2021;89(S1):20-22

Contents available at PubMed www.cirugiaycirujanos.com 
vólvulo pancreático se produce cuando el tejido pancreático gira sobre el ligamento esplenorrenal durante la torsión esplénica. Son pocos los casos de bazo errante asociado a vólvulo pancreático publicados en la literatura y ninguno asociado a una hernia lumbar ${ }^{1-4}$.

Describimos el caso de un bazo errante que se presentó como infarto esplénico y vólvulo pancreático, en asociación con una hernia de Grynfelt-Lesshaft y antecedente de traumatismo abdominal.

\section{Caso clínico}

Mujer de 43 años con antecedente de laparoscopia diagnóstica 5 años antes debido a un accidente automovilístico, en la que se evidenció una lesión hepática de grado II. Acude a consulta de urgencias por dolor abdominal de 1 semana de evolución. Se encontró taquicárdica, con una tumoración palpable en el mesogastrio de $20 \mathrm{~cm}$, móvil, dolorosa, sólida. También se evidenció aumento de volumen en la región lumbar derecha, reducible, la cual refiere apareció semanas después del traumatismo sufrido 5 años antes. La tomografía computarizada contrastada muestra el bazo migrado al mesogastrio, con esplenomegalia, torsión del hilio esplénico hacia el lado izquierdo, datos de infarto esplénico, vólvulo pancreático y una hernia lumbar del triángulo superior derecho de contenido intestinal sin datos de complicación (Fig. 1). Se realiza laparotomía exploradora, en la que se observa un bazo de gran tamaño, con doble rotación sobre el hilio y el ligamento gastroesplénico, causando un vólvulo de la porción distal de la cola del páncreas que condiciona isquemia. Se realizaron esplenectomía, pancreatectomía distal y herniorrafia con puntos simples, cerrando el defecto de la fascia transversal, afrontando el oblicuo interno y el cuadrado lumbar, y se colocó una malla de polipropileno con recubrimiento de hidrogel bioabsorbible intraperitoneal (Fig. 2). La paciente curso el posoperatorio sin complicaciones.

\section{Discusión}

La presentación clínica del bazo errante varía desde un hallazgo incidental, una tumoración abdominal y hasta un abdomen agudo ${ }^{5,6}$. El $67 \%$ de los pacientes se presentan con una masa abdominal en la exploración física ${ }^{7}$. El dolor abdominal puede ser crónico intermitente o agudo intenso, debido a la torsión del pedículo esplénico que causa congestión
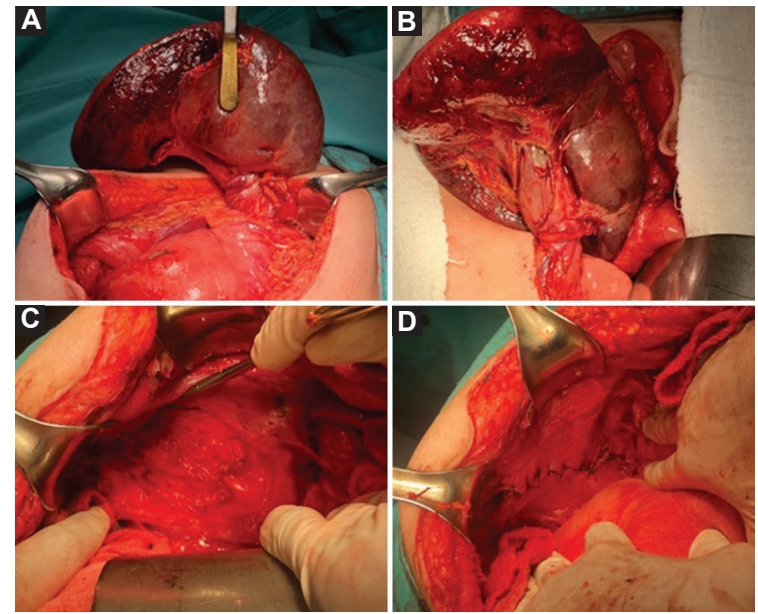

Figura 1. A: bazo de gran tamaño en posición anterior, con áreas hipodensas sugestivas de infarto. Defecto herniario en el triángulo lumbar superior con contenido intestinal, sin datos de complicación. B: corte coronal en el que se aprecia una hernia lumbar. C: vólvulo de la cola del páncreas. D: «signo del giro", patognomónico de torsión de hilio esplénico.
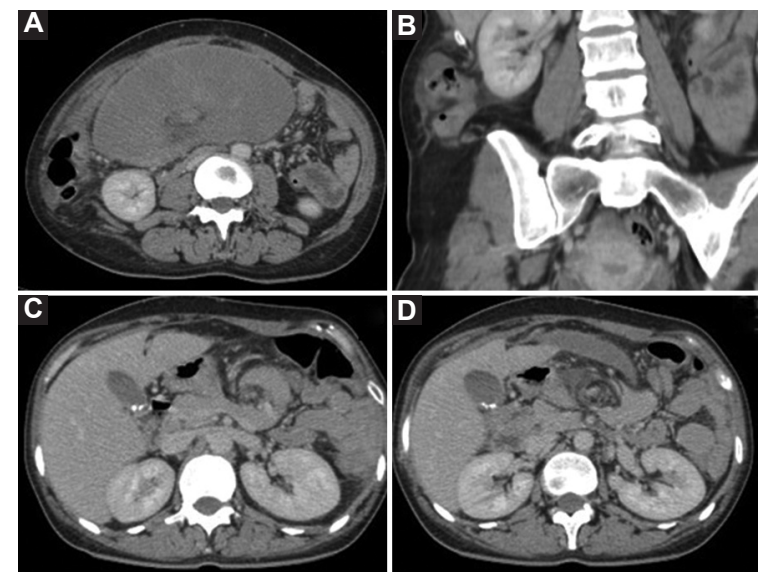

Figura 2. A-B: se aprecian el bazo, el hilio esplénico y el ligamento gastroesplénico con doble rotación hacia la izquierda. C-D: defecto herniario lumbar antes y después de la reparación.

esplénica, tensión capsular e isquemia, hasta progresar al infarto esplénico ${ }^{7,8}$. La tomografía computarizada contrastada es el estudio de elección, pues permite visualizar la posición del bazo y evidenciar el «signo de giro» (Fig. 1 D), específico de la torsión del hilio esplénico'. También detecta si hay compromiso de estructuras adyacentes, como en este caso, en el que se apreció un vólvulo pancreático (Fig. 1 C), y de igual manera afecciones asociadas o de otros órganos, como la hernia lumbar concomitante (Fig. $1 \mathrm{~A}$ y B). El tratamiento quirúrgico del bazo errante depende del contexto clínico y puede variar 
desde solo esplenopexia hasta esplenectomía en caso de infarto esplénico. Si existe compromiso de la cola del páncreas, la pancreatectomía distal es el procedimiento de elección ${ }^{1,3,8,10,11}$. La asociación de bazo errante infartado con afección de otras estructuras es inusual, por lo que encontrar un vólvulo pancreático es sumamente raro ${ }^{10,11}$. La estrecha relación del hilio esplénico y el páncreas facilita que la torsión del bazo sobre su eje vascular condicione movilización de la cola del páncreas, tal como ocurrió en este caso, en el que el ligamento gastroesplénico giró sobre el eje vascular atrapando junto a los vasos esplénicos un segmento de la cola del páncreas, causando el vólvulo pancreático, el infarto de la cola pancreática y el infarto esplénico, con necesidad de realizar una resección parcial de la cola del páncreas y una esplenectomía.

La hernia de Grynfelt-Lesshaft se define como una protrusión de los contenidos intraperitoneales o extraperitoneales a través de un defecto en la pared abdominal posterolateral en el triángulo lumbar superior, y fue descrita por Lesshaft en 1870 y Grynfelt en 1886. Se han reportado alrededor de 300 casos en la literatura ${ }^{12}$. En lo que respecta a su tratamiento, se recomienda realizar plastia con cierre del defecto herniario y colocación de algún tipo de malla ${ }^{12,13}$. En nuestro caso, la plastia se realizó por vía transabdominal, con el cierre del defecto y la colocación de la malla de doble capa en el mismo tiempo quirúrgico, lo cual no es la conducta habitual, pero dado el caso y la realización de una laparotomía media para resolver el bazo errante se optó por hacerlo a través de este mismo abordaje, obteniendo buenos resultados y sin necesidad de realizar un segundo tiempo quirúrgico. Únicamente se han reportado dos casos de bazo errante con vólvulo pancreático en los que se realizó resección de la cola del páncreas ${ }^{11,14}$. Es evidente la relación que existe entre el traumatismo y la aparición de la hernia, pero no está tan clara la relación entre el bazo errante y el traumatismo previo o el procedimiento quirúrgico. Es importante un alto índice de sospecha en pacientes con esta afección para brindar un tratamiento adecuado según la presentación clínica.

\section{Agradecimientos}

Los autores agradecen al personal involucrado en el servicio de cirugía general del Hospital Universitario Dr. José Eleuterio González por ofrecer todo lo necesario para llevar a cabo este trabajo.

\section{Responsabilidades éticas}

Protección de personas y animales. Los autores declaran que para esta investigación no se han realizado experimentos en seres humanos ni en animales.

Confidencialidad de los datos. Los autores declaran que han seguido los protocolos de su centro de trabajo sobre la publicación de datos de pacientes.

Derecho a la privacidad y consentimiento informado. Los autores han obtenido el consentimiento informado de los pacientes y/o sujetos referidos en el artículo. Este documento obra en poder del autor de correspondencia.

\section{Financiamiento}

No se requirió ningún tipo de financiamiento para la realización de este trabajo.

\section{Conflicto de intereses}

Ninguno de los autores presenta conflicto de intereses.

\section{Bibliografía}

1. Asafu Adjaye Frimpong G, Aboagye E, Ayisi-Boateng NK, Antwi K, Bawuah KA, Coleman NE, et al. Concurrent occurrence of a wandering spleen, organoaxial gastric volvulus, pancreatic volvulus, and cholestasis - a rare cause of an acute abdomen. Radiol Case Rep. 2019;14:946-51.

2. Aswani Y, Anandpara KM, Hira P. Wandering spleen with torsion causing pancreatic volvulus and associated intrathoracic gastric volvulus. An unusual triad and cause of acute abdominal pain. JOP J Pancreas. 2015;16:78-80.

3. Flores-Ríos E, Méndez-Díaz C, Rodríguez-García E, Pérez-Ramos T. Wandering spleen, gastric and pancreatic volvulus and right-sided descending and sigmoid colon. J Radiol Case Rep. 2015:9:18-25.

4. Gorsi U, Bhatia A, Gupta R, Bharathi S, Khandelwal N. Pancreatic volvulus with wandering spleen and gastric volvulus: an unusual triad for acute abdomen in a surgical emergency. Saudi J Gastroenterol. 2014;20:195.

5. Magowska A. Wandering spleen: a medical enigma, its natural history and rationalization. World J Surg. 2013;37:545-50.

6. Maestre-Serrano RY, Acevedo-Reyes H, Cohen-Ríos S, Álvarez-Jiménez J. Bazo errante gigante con torsión del pedículo e infarto esplénico: reporte de un caso. Rev Cir. 2019;71:257-60.

7. Soleimani M, Mehrabi A, Kashfi A, Fonouni H, Büchler MW, Kraus TW. Surgical treatment of patients with wandering spleen: report of six cases with a review of the literature. Surg Today. 2007;37:261-9.

8. Fonseca AZ, Ribeiro M, Contrucci O. Torsion of a wandering spleen treated with partial splenectomy and splenopexy. J Emerg Med. 2013:44:e33-6.

9. Raissaki M, Prassopoulos P, Daskalogiannaki M, Magkanas E, Gourtsoyiannis N. Acute abdomen due to torsion of wandering spleen: CT diagnosis. Eur Radiol. 1998;8:1409-12.

10. Seif Amir Hosseini A, Streit U, Uhlig J, Biggemann L, Kahl F, Ahmed S, et al. Splenic torsion with involvement of pancreas and descending colon in a 9-year-old boy. BJR Case Rep. 2019;5:20180051.

11. Sheflin JR, Lee C, Kretchmar K. Torsion of wandering spleen and distal pancreas. Am J Roentgenol. 1984;142:100-1.

12. Piozzi GN, Cirelli R, Maino MEM, Lenna G. Management criteria of Grynfeltt's lumbar hernia: a case report and review of literature. Cureus. 2019;11:e3865.

13. Loukas M, El-Zammar D, Shoja MM, Tubbs RS, Zhan L, Protyniak B, et al. The clinical anatomy of the triangle of Grynfeltt. Hernia. 2008;12:227-31.

14. Choudhary R, Ghazanfari A. Wandering spleen with pancreatic volvulus and colonic obstruction in an elderly patient. Int $\mathrm{J}$ Case Rep Images. 2012;3:15. 